\title{
Regions on Nucleocapsid Protein of Newcastle Disease Virus that Interact with its Phosphoprotein
}

\begin{abstract}
The nucloecapsid (NP) and phosphor-(P) proteins of paramyxoviruses are involved in transcription and replication of the viral genome. An in vitro protein binding assay was used to investigate the regions on NP protein that interact with the P protein of Newcastle disease virus (NDV). Truncated NP mutants were first immobilized on a solid phase and then interacted with radio-labelled [35S]-P protein synthesised in rabbit reticulocyte. The interaction affinity was quantitated by measuring the radioactivity that was retained on the solid phase. Using the approach, a highly interactive region was identified to be resided at the first 25 amino acids of NPN-terminal. The interaction between these two proteins remained strong even with the removal of 114 amino acids from the $\mathrm{C}$-terminal end might have another contact region for $\mathrm{P}$ protein, which is not as critical as the N_terminal end. The interaction regions mapped in this study are significantly different from the other two paramyxoviruses: Sendai and measles viruses in which the C-termini of their NP proteins play an important role in binding to the $\mathrm{P}$.
\end{abstract}

